\title{
An incentive model for the overlearning reversal effect'
}

John Theios and Dennis Blosser UNIVERSITY OF TEXAS

\begin{abstract}
A theoretical model is proposed for the interaction of the effect of number of training trials and magnitude of reward on speed of reversal learning. The model was tested by an experiment involving 2 levels of reward magnitude and 4 degrees of training. An overlearning reversal effect was obtained under large reward but not with small reward. The data from the 8 groups of rats were well within chance limits of the quantitative predictions from the model.
\end{abstract}

\section{Problem}

Theios \& Brelsford (1964) and Theios \& Blosser (in press) have suggested that the controversy over the existence of the overlearning reversal effect (ORE) can be resolved by consideration of the variable of magnitude of reward. By and large, the ORE tends to be obtained when a large reward magnitude is used, and not obtained when a small magnitude is used.

The purpose of the present experiment is to provide a test of quantitative predictions derived from a theoretical model suggested by Theios \& Brelsford (1964) and Theios \& Blosser (in press).

Briefly the model assumes that:

1. Habit strength is an increasing exponential function of the number of training trials on the original task:

$$
\mathrm{H}_{\mathrm{n}}=1.00-(1.00-\mathrm{a})^{\mathrm{n}-1}
$$

where $n$ represents the number of trials of original training, $H_{n}$ represents habit strength after $n$ trials, and $a$ is rate parameter, $0<a<1.00$.

2. Incentive motivation is an increasing exponential function of the number of appetitively rewarded training trials to the original goal. The asymptote of the function is directly related to the magnitude of reward, and the rate of the function is directly related to the number of rewards experienced at the original goal before training begins:

$$
\mathrm{K}_{\mathrm{N}}=\mathrm{M}-\mathrm{M}(1.0-\mathrm{b})^{\mathrm{N}-1}
$$

where $\mathrm{N}$ represents the number of rewarded trials during original training, $\mathrm{K}_{\mathrm{N}}$ represents incentive motivation after $\mathrm{N}$ rewarded trials, $\mathrm{M}$ is the asymptote of the function, $b$ is the rate parameter, $0<M<1.00$, and $0<\mathrm{b}<\mathrm{a} / \mathrm{M}$.

3. The effects of habit strength and incentive motivation of the original response interact in such a way during extinction or reversal that the expected number of trials to an extinction or reversal criterion is equal to:

$$
\mathrm{E}\left(\mathrm{R}_{\mathrm{n}, \mathrm{N}}\right)=\mathrm{c}\left(\mathrm{H}_{\mathrm{n}}-\mathrm{K}_{\mathrm{N}}\right) .
$$

$E\left(R_{n, N}\right)$ represents the expected trials to criterion after $\mathrm{n}$ original training trials and $\mathrm{N}$ rewards and c is a constant which can be taken to equal 100 to establish a centigrade scale.

The assumptions of the model have obvious referents in the behavior systems of Amsel (1962) and Spence (1960), particularly with respect to the concept of frustrative nonreward. Habit strength $\left(\mathrm{H}_{n}\right)$ is an increasing function of the number of original training trials. Number of responses to a reversal or extinction criterion is an increasing function of $\mathrm{H}_{\mathrm{n}}$. Incentive motivation $\left(\mathrm{K}_{\mathrm{N}}\right)$ is an increasing function of the number, size, and quality of rewards. The magnitude of disruptive frustrative responses $\left(r_{f}\right)$ occurring on trials subsequent to nonreward is an increasing function of $\mathrm{K}_{\mathrm{N}}$. Number of responses to a reversal or extinction criterion is inversely related to the magnitude of the frustrative responses, and hence, inversely related to $\mathrm{K}_{\mathrm{N}}$. Thus, the function relating responses to a reversal or extinction criterion to $\mathrm{H}_{\mathrm{n}}$ and $\mathrm{K}_{\mathrm{N}}$ must depend on their difference as postulated in Equation 3.

\section{Method}

The Ss were 80 naive male albino rats approximately 90 days old. The apparatus and procedure were similar to that reported in detail in Experiment II of Theios \& Blosser (in press). The apparatus was a 5 in wide T-maze with 10 in long start and goal boxes. The interior choice section of the maze had a 10 in stem and a 15 in cross member. One goal box was painted black, the other white, and the rest of the maze was grey. For each $\mathrm{S}$ reward was placed always in the left or always in the right goal box during training and in the opposite goal box during a reversal test. The experiment was a 2 by 4 factorial design. The Ss were rewarded with either two $.045 \mathrm{gm}$ Noyes pellets or $20 \mathrm{sec}$. free access to wet mash. The Ss were given either 10,40 or 120 free choice trials, or 110 direct goal box placements followed by 10 free choice trials before the reward was shifted to the opposite side of the maze. On error trials $\mathrm{S}$ was detained in the empty goal box for 5 sec. During the first 110 trials each Placed S was yoked to an 120 trials S such that he was placed in the goal box which his partner chose and was rewarded if his partner received reward. During reversal learning each $\mathrm{S}$ received the same size reward as during original learning. Each $\mathrm{S}$ was run to a reversal criterion of 10 consecutive correct responses. 


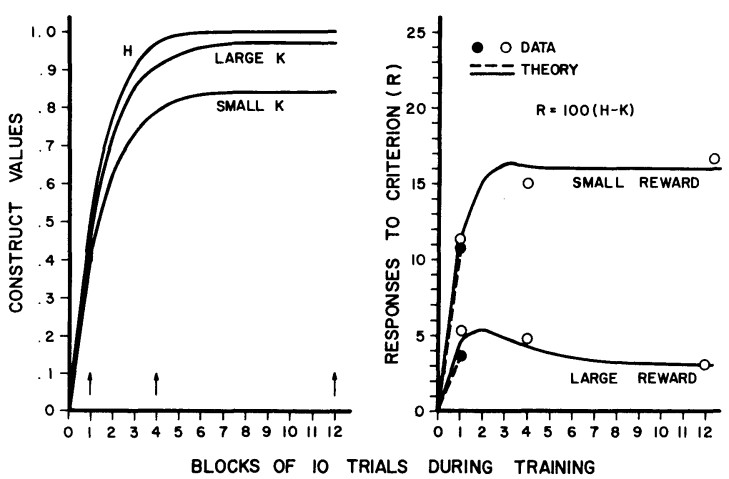

Fig. 1. The left half of the figure presents the theoretical growth curves for $\mathrm{H}_{\mathrm{n}}$ and $\mathrm{K}_{\mathrm{N}}$. The arrows indicate the construct values for the various conditions of the experiment. The right half of the figure presents the obtained mean numbers of responses to the reversal criterion along with the predictions from the model. The predictions for the Placed groups are designated by dashed lines.

\section{Quantitative Predictions and Results}

Since the rate parameter a of Equation 1 for habit strength is independent of magnitude of reward, all 8 groups will have the same value of a. The two Placed groups will have the same rate parameter, b', for $\mathrm{K}_{\mathrm{N}}$ of Equation 2, which should be larger than the rate parameter, $b$, for the rest of the groups. The asymptotes of $\mathrm{K}_{\mathrm{N}}$ will be $\mathrm{M}$ for large reward and $\mathrm{m}$ for small reward. The numerical values of the parameters were estimated with a high speed computer which minimized the squared deviations of obtained mean trials to criterion from predicted $E\left(R_{n, N}\right)$. The estimates are $\mathrm{a}=.084, \mathrm{~b}^{\prime}=.078, \mathrm{~b}=.076, \mathrm{M}=.969$, and $\mathrm{m}=.840$. The theoretical growth curves for $\mathrm{H}_{\mathrm{n}}$ and $\mathrm{K}_{\mathrm{N}}$ are given in the first half of Fig. 1. The obtained mean number of responses to the reversal criterion are presented in the second half of Fig. 1 along with the theoretical predictions from the model. A goodness of fit analysis indicated that the predictions are well within chance limits of the data points, $F(3,72)=.15 ; p<.50$.

\section{Diseussion}

A number of qualitative predictions which follow from the model can be seen by inspecting Fig. 1 or families of similar curves. A few of the more salient predictions are:
1. There is an interaction between magnitude of reward and degree of training such that an ORE is more likely to be obtained when large reward is used than when small reward is used.

2. Within any reward level, whether or not an ORE is obtained will be determined by the number of training and overtraining trials used and by the values of the parameters of Equations 1 and 2. For example, with large reward a failure to obtain the ORE could occur by (a) having the criterion and overlearning groups straddle the maximum of the function given in Equation 3, or (b) by giving criterion Ss so many trials that they are so far past the maximum of the $\mathrm{H}_{\mathrm{n}}-\mathrm{K}_{\mathrm{N}}$ function that both the criterion and overlearning Ss are on the relatively flat portion of the curve.

3. Within any reward level, the relationship between number of responses to criterion and number of training trials should be monotone increasing only over a relatively small number of training trials.

4. With training trials equated, the number of responses to criterion will be a decreasing function of magnitude of reward.

The majority of the studies in the overlearning literature can be accounted for by this model. In light of the present research, there is a simple reason for the conflicting results reported by earlier investigators and the ensuing controversy over the existence of the ORE. Using just two groups, experimenters were trying to explore a possibility space which can be described as a nonmonotonic surface. With this state of affairs any result is possible with only two groups, depending upon the parameters of the experiment.

\section{References}

AMSEL, A. Frustrative nonreward in partial reinforcement and discrimination learning: Some recent history and a theoretical extension. Psychol. Rev., 1962, 69, 306-328.

SPENCE, K. W. Behavior theory and learning. Englewood Cliffs, N. J.: Prentice Hall, 1960.

THEIOS, J., \& BLOSSER, D. The overlearning reversal effect and magnitude of reward. J. comp. physiol. Psychol., in press. THEIOS, J., \& BRELSFORD, J. Overlearning-extinction effect as an incentive phenomenon. J. exp. Psychol., 1964, 67, 463-467. Note

1. This research was supported by PHS Research Grant HD 00950 from the National Institute of Child Health and Human Development. 